\title{
O raciocínio geográfico e as chaves de leitura da Covid-19 no território brasileiro
}

\author{
RAUL BORgES GUIMARÃES, ${ }^{I}$ RAFAEL DE CASTRO CATÃO, II \\ OSÉIAS DA SILVA MARTINUCI, III EDMUR AZEVEDO PUGLIESI IV \\ e PATRICIA SATURI SILVESTRE MATSUMOTO ${ }^{\mathrm{V}}$
}

\section{Introdução}

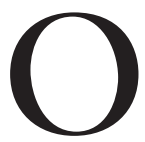

PRESENTE artigo foi concluído no dia em que o Brasil completava 100 dias desde o primeiro caso confirmado de Covid-19. Durante esses dias, pudemos acompanhar o crescimento acentuado de informações sobre casos da doença e suas repercussões, com dados atualizados diariamente, seja no nível municipal, seja no estadual, seja no regional, seja no nacional. Como parte de um grande esforço coletivo de compreensão da situação de emergência em saúde pública gerada pela pandemia da Covid-19 no Brasil, foi possível acessar dados livres em diferentes plataformas colaborativas, o que indicaria para a democratização dos mapeamentos em alta velocidade, e a possibilidade de disponibilizar ao público em geral o acompanhamento das ações de enfrentamento quase em tempo real.

Contudo, tais iniciativas nem sempre foram acompanhadas de uma reflexão acerca da qualidade da informação produzida, como é o caso da análise espacial a partir de diversos processos de mapeamento da Covid-19. Em tempos de globalização, a tarefa de análise desse processo de produção de informações tornou-se ainda mais árdua, pois o espaço geográfico está sujeito e é constituído, simultaneamente, por um crescente número de relações de toda ordem. Tendo grande consciência disso, Milton Santos $(2008$, p.17) afirmou em uma das suas últimas obras que "vivemos num mundo confuso e confusamente percebido". Lembra-nos esse autor que "a complexidade do mundo é marcada pelo aumento vertiginoso de objetos e pela infinidade de relações que esses objetos sujeitam os processos sociais e espaciais" (ibidem, p.171). É o pressuposto da totalidade que fornece o caminho necessário para a compreensão dos eventos e das situações geográficas específicas. Cada coisa no mundo tem seu sentido dado pelo conjunto de relações ao qual está sujeito e do qual resulta (Kosik, 1989; Silveira, 1999). A totalidade que, ao mesmo tempo, evita imagens simplificadoras do 
mundo fornece os fundamentos necessários para apreender as principais estruturas que governam e dão sentido aos eventos, o que não seria diferente diante da rápida difusão espacial do novo coronavírus.

Sabemos que o registro de casos confirmados da Covid-19 no Brasil é muito deficiente e com grande proporção de subnotificações, além dos casos assintomáticos e oligossintomáticos (Mellan et al., 2020). Tal dificuldade já era esperada, uma vez que é preciso definir com maior precisão indicadores de incidência e de transmissibilidade do novo coronavírus (Baud et al., 2020). Para enfrentar tais dificuldades, diversos grupos de pesquisa têm se utilizado de eventos sentinelas, como as internações por Síndromes Respiratórias Agudas Graves (SRAG), ou se baseado na análise de óbitos por Covid-19 para propor procedimentos técnicos adequados para a análise espacial da ocorrência da Covid-19 no Brasil, assim como relacionar essa ocorrência de casos e a distribuição espacial de óbitos por Covid-19 às condições socioambientais de diferentes cidades brasileiras (Rodrigues et al., 2020; Alcântara et al., 2020).

Tomando como referência essa compreensão dos eventos em saúde em suas conexões com a totalidade, ressaltamos que a difusão espacial da Covid-19 não se trata de um problema de saúde pública nos mesmos moldes que outras pandemias trouxeram, mas de um desafio a ser enfrentado cada vez mais relevante no mundo globalizado em que vivemos. Assim, alteraram-se as escalas da vida e da economia, ampliaram-se os cruzamentos impostos por um mundo mais complexo, e por isso se torna necessário transformar nosso olhar para novos problemas.

Também sabemos que, diante dessa nova pandemia, o desenvolvimento de metodologias diagnósticas e prognósticas, por exemplo, a proposta de vigilância epidemiológica baseada em modelos espaço-temporais, é essencial à rápida resposta demandada pela saúde pública. Segundo MacEachren (2004), a análise espacial com base em ferramentas quantitativas contribui para evidenciar a importância do arranjo espacial na distribuição do fenômeno mapeado que, no caso específico do presente trabalho, refere-se aos casos confirmados da Covid-19 no território brasileiro. Mas o aporte dos modelos espaciais obtidos da análise geoespacial não é um fim em si mesmo (Rojas, 2008). Ao contrário do mapa ser resultado da análise, ele deve ser considerado como base na identificação de estruturas espaciais elementares (Brunet, 2001), chaves de leitura da disseminação da Covid-19 no território e o caminho metodológico estabelecido por uma espécie particular de raciocínio científico: o raciocínio geográfico. Veremos que essa forma de pensar a disseminação da Covid-19 no Brasil, a partir de conceitos e procedimentos metodológicos da geografia, foi desenvolvido nos últimos 100 anos e conta com a contribuição de obras clássicas dessa disciplina, com a aplicação de modelos matemáticos e geotecnologias para a correlação e a síntese de grande volume de informações espaciais. Apresentaremos sucintamente a seguir quais são esses princípios científicos formulados pela geografia para, em seguida, discutir os passos metodológicos necessários para uma melhor compreensão da pandemia, considerando os dados dessa doença no Brasil. 


\section{A contribuição dos clássicos da geografia e áreas afins}

Em meados da década de 1920 o geógrafo francês Maximillien Sorre propôs uma forma de análise integrada e relacional para a compreensão das doenças no espaço geográfico. Para isso, ele denominou complexo patogênico as associações estáveis (no tempo e no espaço) entre os entes da cadeia epidemiológica (vetores, reservatórios, agentes patológicos e o ser humano) e o espaço geográfico, que conformaria uma extensão territorial (área de ocorrência) de uma determinada doença. A existência do complexo territorialmente delimitado conduziria a produção de doenças. Dessa forma, a área de localização de determinada doença daria pistas para a compreensão de fatores socioambientais relacionadas à sua ocorrência. Para ele, as doenças vetoriais especialmente as transmitidas por artrópodes (Sorre, 1933) são menos ubíquas, com condições de vida mais influenciadas pelo meio geográfico e, dessa forma, mais localizadas. Doenças de transmissão pessoa a pessoa têm o ecúmeno como probabilidade de extensão, desde que haja as condições de circulação necessárias.

Estabelecendo, portanto, como princípios do raciocínio as relações entre a localização dos eventos, a extensão desse fenômeno e suas conexões com outros fenômenos de interesse social, Max Sorre destaca a importância metodológica da produção cartográfica. Segundo ele, "os primeiros objetivos de qualquer mapa são localizar os fenômenos, o local onde ocorreram; segundo determinar as áreas de extensão desses fenômenos; e terceiro marcar as variações de intensidade dentro destas áreas. A representação do movimento ocorre somente depois" (Sorre, 1978, p.239). Como Sorre (1933) postula que o complexo patogênico seria como uma entidade biológica de nível superior, com início, desenvolvimento e extinção, além de movimentos de expansão e de contração, sua obra permitiu abordar grande variedade de doenças infecciosas e parasitárias, como nos estudos da dengue e das leishmanioses (Guimarães et al., 2018).

Cabe questionar se esse conceito formulado por Marx Sorre na década de 1930 seria suficiente para descrever e compreender os fatos e processos atuais, como é o caso da primeira pandemia do capitalismo globalizado: a pandemia da Covid-19. Diante dessa indagação, uma das formas de fortalecer nosso arcabouço teórico não seria apenas de abandonar nossos conceitos disciplinares e simplesmente formular outros. É preciso fazer a releitura dos clássicos e reformular os conceitos originais diante dos novos desafios impostos, como procurou fazer Rojas (2019). Para ela, o complexo poderia definir-se na atualidade como "entidade bio psico social de ordem superior que resulta da dependência mútua entre componentes e processos biológicos, políticos, econômicos, culturais e ambientais que participam da produção de determinados problemas de saúde" (Rojas, 2019, p.16).

Temos aí um ponto de partida de método. Numa primeira instância, os dados mapeados, devidamente localizados no espaço - e compreendidos em sua extensão e conexão com outros fenômenos mapeados, devem ser analisados tendo em vista uma ordem espacial superior, uma estrutura de ordem política e 
econômica que explica a produção social da doença. No que se refere ao processo de difusão espacial da Covid-19, o novo coronavírus, transmitido de pessoa a pessoa (sem intermediação de vetores ou hospedeiros) origina-se na capital Wuhan, província chinesa de Hubei, onde existe um importante bub de circulação mundial e possui uma população de mais de 10 milhões de pessoas (Canadá, 2020). Houve uma epidemia em seu local de origem e após o bloqueio começou a diminuir sua importância enquanto um novo centro surgia na Europa e, posteriormente, nos Estados Unidos, tomando dimensões de uma pandemia.

Como esse processo resultou de um espalhamento de um novo coronavírus no espaço e ao longo do tempo, esse fenômeno nos remete a outras abordagens clássicas da geografia que procuram explicar essa propagação, com base nos estudos de Torsten Hägerstrand (1962; 1968) sobre modelos de difusão de inovações. Graças a esses estudos o tema da difusão espacial foi exaustivamente trabalhado, especialmente durante as décadas de 1960-1980 pela corrente da Nova Geografia, que desenvolveu técnicas e modelos para interpretação desse processo. Esse geógrafo sueco, e seu grupo na Universidade de Lund, conseguiu modelar o processo de difusão de inovações, dando enfoque espacial e um embasamento matemático e estatístico. Posteriormente, devido ao direcionamento econômico e locacional, essa obra aporta na Escola de Washington nos Estados Unidos, no final da década de 1960, com uma grande aceitação pela Nova Geografia. A difusão espacial é entendida como um processo no qual um fenômeno, item ou ideia sai de um, ou poucos pontos restritos, e aumenta sua área. Esse movimento é indissociável no tempo e espaço.

A importância dos trabalhos de Hägestrand após várias análises de processos de difusão (como de carros, tratores, telefones e técnicas agrícolas) consiste em: 1) conceituação do processo de difusão de inovação; 2) criação de um arcabouço teórico e metodológico para essa análise; e 3) identificação de regularidades empíricas da difusão de inovações (Brown, 1981). A obra de Hägerstrand é um marco também pela sistematização de regularidades empíricas relacionadas a difusão. A primeira é o gráfico em forma de ' $S$ ' ou curva logística quando plotado com o tempo e a frequência cumulativa de adotantes de determinada inovação. A segunda regularidade empírica é denominada Efeito de Hierarquia, consiste no fato de que "no sistema urbano, a difusão é esperada a proceder dos centros grandes para os pequenos" (Brown, 1981, p.21, tradução nossa). O efeito de Hierarquia está presente em vários estudos de difusão, que as cidades maiores e com hierarquias urbanas mais elevadas recebem as inovações primeiro e as difundem na rede urbana. A conexão entre as metrópoles de ordem hierárquica mais alta em todo mundo permite a circulação dessas inovações primeiramente nesse circuito, apesar de que atualmente já temos outras formas de ligação entre centros, com menos rigidez que na década de 1960.

Se pensarmos essas duas regularidades juntas, podemos imaginar que no início da curva logística as cidades maiores e mais centrais na rede urbana serão 
as primeiras a ter adotantes, já as cidades menores e áreas rurais estariam no final do processo.

A terceira regularidade consiste no Efeito de Vizinhança ou de Contágio, em "uma hinterland ou uma única cidade a difusão é esperada proceder em uma forma de onda para fora do centro urbano primeiro atingindo lugares próximos ao invés de distantes" (Brown, 1981, p.21).

Mais adiante, o presente artigo detalha a análise espacial efetuada da difusão da Covid-19 no território brasileiro como um caminho metodológico capaz de fornecer respostas em tempo real à saúde pública brasileira. Estamos convictos de que existem diferentes situações epidemiológicas no Brasil, assim como muitas disparidades regionais (Fortaleza, 2020). Por conseguinte, são necessários modelos espaço-temporais e estudos epidemiológicos cujos achados embasem aplicação imediata por meio de modelos matemáticos clássicos e análises de Geografia da Saúde, como descritos acima.

Contudo, apesar da enorme importância do uso da análise quantitativa para a identificação dessas regularidades no processo de difusão de doenças infectocontagiosas, como a Covid-19, tais procedimentos não seriam suficientes para a identificação de estruturas espaciais elementares como chaves de leitura da disseminação da doença no território brasileiro. Por isso, entendemos ser necessário, além da etapa de análise exploratória dos dados, avançar na modelização geográfica e na visualização cartográfica, o que tem como referência outros autores clássicos da geografia, como Brunet e Berry.

Não nos esqueçamos de que o espaço geográfico, objeto de estudo do geógrafo, só pode ter seu sentido apreendido no nível da totalidade. Considerando essa problemática, o geógrafo francês Roger Brunet (2001) propôs uma metodologia que fornece subsídio para a identificação das principais estruturas do espaço geográfico. De acordo com Brunet, a identificação começa basicamente pela consideração do complexo espacial, ou seja, do conjunto de relações existentes, e termina quando o ganho marginal se torna muito baixo. Pode-se, com isso, separar as estruturas fortes, que dão sentido a uma situação geográfica ou a um evento qualquer, e as estruturas contingentes, ou seja, aquelas pouco significativas como doadoras de sentido (Brunet, 2001). Nesses termos, vê-se que o sentido reside nas relações. O desafio, portanto, é identificar as estruturas que estão mais fortemente ligadas umas às outras e que se condicionam mutuamente. Essa ideia que remete aos fundamentos axiológicos da teoria de Brunet evidencia a relação entre processos espaciais, que implica a indissociabilidade do tempo, e as formas espaciais, aquilo que é um fixo territorial e resiste às mudanças (e isso não significa que elas não mudem, mas que tendem a ser mais duráveis). A indissociabilidade entre fixos territoriais e processos espaciais levou Milton Santos (2002) à ideia de inércia dinâmica, pois os primeiros condicionam tão fortemente os segundos que as estruturas espaciais resultantes são muito similares. 
Adicionalmente, Brunet argumenta que modelização não corresponde à simplificação, seja ela geométrica ou semântica, um mal-entendido muito frequente. Para ele:

É um erro profundo confundir simplificação e modelização, em especial em geografia, na qual a simplificação é confundida com a "generalização" de contornos no sentido de cartógrafos. [...] A modelização assume hipóteses que dependem da natureza e da situação do objeto geográfico estudado. Ela os associa e os empurra aos seus limites; constrói, desconstrói, reconstrói; ele passa por uma série de interações entre dedução e indução. Seus primeiros desenhos, se houver, estão no domínio do abstrato; pouco a pouco eles vão se aproximando da complexidade da realidade, que nunca é simplificada. [...] A única decisão estratégica é escolher quando parar o processo, que deixa nas sombras o que é considerado incidental, mas é uma questão comum a toda atividade intelectual e que respeita as leis da lógica. (Brunet, 2000, p.28, tradução nossa)

Além de não corresponder à simplificação, uma vez que busca a complexidade de situações geográficas, um modelo territorial nunca é definitivo (Théry, 2004). Sendo as estruturas territoriais resultado do trabalho da sociedade, elas estão evoluindo permanentemente, o que implica rever os modelos com frequência. Esse é um pressuposto fundamental, ou seja, que os fixos territoriais ou os sistemas de engenharia, como denominaram Milton Santos e Maria Laura Silveira (2001), são essencialmente sociais, pois, ao produzi-los, o homem funde-se com o objeto do seu trabalho, seja porque tem trabalho morto internalizado, seja porque eles se tornam imediatamente interdependentes.

Uma das contribuições importantes do geógrafo Milton Santos por nós utilizadas para discutir o raciocínio geográfico da Covid-19 são as estruturas espaciais representadas pelos espaços luminosos e opacos, que remetem a uma análise da densidade de informação, tecnologia e conhecimento no território brasileiro, que tem como consequência uma seletividade espacial por parte do capital. Partes do território dotadas de informação "competem vantajosamente com as que deles não dispõe" (Santos, 2002, p.194). Destaca-se que os territórios de alta densidade técnica-informal tornam-se mais aptos a atrair investimentos econômicos, capitais, tecnologia e organização, logo, são denominados territórios luminosos. Contraditoriamente, os territórios não dotados dessas características são denominados territórios opacos (Santos, 2002).

O modelo, ao representar essas estruturas, não está preocupado com simplificação ou centrado na comunicação, mas objetiva captar um sistema de relações para fins de investigação e interpretação. Ao representar as estruturas fortes, que Brunet chamou de "coremas", o modelo ajuda a dar sentido a eventos geográficos, como é o caso da Covid-19, e fornecer condições para pensar e agir sobre o território.

Uma forma de se sistematizar essa compreensão da dinâmica espacial da Covid-19 remonta às abordagens para análise regional criadas por Berry (1964), 
uma síntese a partir da relação sítio-situação. A partir do par dialético, estabelece-se uma matriz geográfica em que se considera o fenômeno espacial e sua inter-relação. Nessa matriz, o sítio é vertical, forma e morfologia. Já a situação é horizontal, refere-se a função, interdependências regionais e conexões entre os lugares, ou as chamadas interações espaciais. A terceira dimensão, o tempo, complexifica o par sítio-situação, uma vez que uma simples característica é observada em um local, com um ponto particular de tempo. Sendo assim, varia-se espacialmente e temporalmente e isso pode ser mapeado e analisado em séries estatísticas e em distribuições de frequência ou espaciais.

Do cruzamento da matriz de Berry, as abordagens são criadas conforme a distribuição espacial do fenômeno, a associação localizada de características no lugar - inventário locacional, pela covariância espacial ou associação espacial de características, pela diferenciação areal, mudanças de distribuição espacial de uma característica, assim como pelas mudanças de características em uma área - observação subsequente; também, pelas mudança da associação espacial de características, de áreas e, por fim, as interações (Berry 1964, tradução nossa).

Adaptando essas abordagens aos dias atuais, podemos implementar as perguntas geoespaciais aos sistemas de informação geográfica (Ferreira, 2013). Elas ajudam a construir uma síntese e organização do pensamento geográfico sobre o tipo de mapeamentos e modelagem espacial que queremos construir. Na análise espacial da Covid-19 a partir do raciocínio geográfico, trata-se de uma forma de criar objetivos cartográficos diante das análises exploratórias traduzidas em perguntas das mais simples às mais complexas (Matsumoto 2019), tais como:

1. Qual área total da ocorrência de casos da Covid-19 no Brasil?

2. Qual é a área total da ocorrência de casos de Covid-19 nos 5.570 municípios brasileiros e no Distrito Federal?

3. Onde ocorrem casos da Covid-19 nas regiões brasileiras (macrorregiões do IBGE e espaços opacos e luminosos) no período $\mathrm{T}_{\mathrm{i}}$ ?

4. Como evoluíram os casos da Covid-19 pelo território brasileiro?

5. Como evoluiu, período a período, a ocorrência de casos da Covid-19 em um conjunto de municípios brasileiros?

6. Qual é o grau de correspondência espacial entre as áreas de ocorrência de casos de Covid-19 e seus fatores de risco, bem como as vulnerabilidades socioespaciais?

7. Dentre o conjunto de todos os municípios, quais são os que apresentam maior semelhança segundo os casos da Covid-19? E de óbitos? Considerar regiões metropolitanas, capitais, cidades médias e interioranas;

8. No período de $T_{i}$, como evoluiu a correspondência espacial entre a ocorrência de casos da Covid-19 localmente?

Essas perguntas geoespaciais constituem um aspecto epistemológico inerente ao processo de mapeamento que seguirá, por sua vez, as etapas da imple- 
mentação da técnica, em que outras questões são colocadas e serão debatidas na próxima seção.

\section{O processo de mapeamento}

Para a identificação das chaves de leitura da Covid-19 no território brasileiro por meio do raciocínio geográfico tomamos como procedimento metodológico central o processo de mapeamento. Com base no método de Sorre (1957), não há como pensar geograficamente o fenômeno da disseminação espacial da Covid-19 sem responder uma pergunta: onde está?, esse é o princípio da localização, o espaço geodésico é importante para uma aproximação da realidade; enfim, conhecer os problemas a partir dos contextos reais. Nesse sentido, propomos aqui uma série de procedimentos, marcados pela análise exploratória dos dados, a modelização espacial e a comunicação cartográfica.

A análise exploratória de dados pode ser considerada desde a implementação dos dados no sistema, utilizando-se de ferramentas básicas de cartografia e dos Sistemas de Informação Geográfica (SIG), até a aplicação de técnicas mais específicas e complexas, com modelos matemáticos e estatísticos - esses procedimentos remontam às perguntas geoespaciais pontuadas acima. Dessa maneira, para se construir esse raciocínio geográfico é preciso dominar técnicas e formas de representação baseados em princípios da cartografia sistemática e temática, que vão desde a noção de trabalhar a distribuição espacial do fenômeno - utilizando-se de sistemas de referência adequados, projeções, técnicas baseadas no tipo de dado, para citar alguns - até sua correlação e associação com diferentes fenômenos/variáveis estudados, envolvendo medidas de dispersão e centralidade com base em procedimentos estatísticos, com o objetivo de avaliar de forma sistemática eventuais padrões espaciais.

Nesse sentido, os principais dados utilizados foram os casos confirmados de Covid-19 no período de 5 de fevereiro a 29 de maio de $2020 .^{1}$ Esses são os dados basais de nossa investigação, constituindo as duas primeiras perguntas geoespaciais. A partir da distribuição dos casos de Covid-19, calculamos taxas, aplicamos técnicas estatísticas e os cruzamos com outros dados, tais como da rede de infraestrutura de transportes terrestres, aéreos, aglomerados subnormais, demarcação das terras indígenas, entre outros.

Para a análise da difusão espacial utilizamos os casos confirmados de Covid-19 nas sedes municipais, assinalando a semana dos primeiros casos confirmados (semanas epidemiológicas de 9 a 22). As sedes sem casos de Covid-19 foram definidas como a semana posterior à última, para preservar o sentido do fluxo. Esse dado foi interpolado por meio da técnica Inverso da Distância Ponderada (do inglês, Inverse Distance Weigthed), um interpolador de efeito local que não extrapola os valores amostrados. A técnica cria uma superfície em formato matricial (raster) em que cada pixel incorpora o valor ponderado dos pontos (no caso as semanas) dos vizinhos em relação direta ao inverso da distância (com um fator de decaimento de 1.5 em relação a distância). Consideram-se, assim, os valores 
estimados para cada ponto, em função da distribuição de um peso inversamente proporcional à distância dos pontos amostrais e os pontos a estimar, de forma com que os valores mais próximos dos pontos têm maior influência do que os valores mais afastados. Dessa forma, criamos uma superfície em que os efeitos de difusão espacial são ressaltados continuamente com variação das semanas dos primeiros casos, possibilitando visualizar o fenômeno temporalmente. Esse mapeamento responde às perguntas geoespaciais de número 3,4 e 5 , em que se insere a questão temporal da Covid-19 no sítio-situação.

Para responder as perguntas geoespaciais de 1 a 7 , utilizamos as técnicas Média do Vizinho Mais Próximo, Superfície de Densidade Kernel, Mapa de Calor, Índice de Moran, Indicador de Associação Espacial Local, e Mapa Coroplético. Para a média do vizinho mais próximo foram considerados todos os pontos que correspondem às localizações das sedes dos municípios brasileiros. Duas superfícies de densidade Kernel foram criadas. Para ambos os casos, o peso atribuído ao cálculo foi a taxa de casos confirmados de modo que os pontos mais próximos do centro da janela móvel do operador recebam um peso maior do que os localizados mais distantes. O mapeamento de densidade de kernel fornece um meio de identificar visualmente os agrupamentos (Câmara et al., 2004a). O tamanho da célula para o modelo foi de 500 metros, valor que se mostrou apropriado para garantir uma superfície suave que contribui no processo de visualização e comunicação da informação cartográfica. Não informamos um raio específico para calcular a superfície, uma vez que o próprio ArcGIS 10.8 disponibiliza o algoritmo de variante espacial conhecido como Silverman's Rule of Thumb, considerado robusto para análises que envolvem outliers (Silverman, 1986). Valores iguais a zero foram removidos do conjunto de dados para garantir maior fidelidade no resultado. Também utilizamos a função Mapa de Calor (Heat Map) disponível no ArcGIS Pro. Foi selecionado o parâmetro de densidade constante, de modo que as áreas por toda a extensão do mapa possam ser comparadas para a mesma escala.

O Índice de Moran, uma expressão computacional do conceito de dependência espacial, foi aplicado para verificar autocorrelação espacial. O prefixo auto indica que a medida de correlação é realizada com a mesma variável aleatória avaliada em locais distintos do espaço (Câmara et al., 2004b). A análise espacial de áreas compreende métodos de análise de dados espaciais cuja localização está associada a áreas que são delimitadas por polígonos. Na conceitualização dos relacionamentos espaciais utilizamos o Inverso da Distância, uma vez que quanto mais próximas as feições estiverem no espaço, maior será a probabilidade de umas influenciarem as outras. Aplicamos o Indicador de Associação Espacial Local (LISA) (Anselin, 1994). O LISA permite estimar valores específicos para cada região e, consequentemente, identificar agrupamentos. Aqui também utilizamos o Inverso da Distância para conceitualizar os relacionamentos entre os dados da Covid-19 distribuídos pelos municípios brasileiros. 
Em seguida, a representação coroplética foi realizada com a técnica de Quebras Naturais, uma vez que não foi detectada a presença de normalidade nos dados. A partir dos resultados obtidos com as duas superfícies de densidade kernel e os agrupamentos detectados a partir do indicador de associação espacial local, foi possível definir um número de classes que melhor representasse o conjunto de dados. Os valores iguais a zero e valores nulos foram removidos para garantir fidelidade no resultado. Uma sequência de cores foi criada para determinar uma representação que partisse de tons escuros para valores mais baixos e tons mais saturados para valores mais elevados. A partir de um processo interativo determinamos um total de dez classes.

Especificamente referente à pergunta geoespacial 6 , que remete às correlações, verificamos a relação existente entre os dados dos casos da Covid-19 e os aglomerados subanormais por meio da correlação de Pearson, baseada na análise geométrica das áreas manipuladas no ArcGIS 10.8. Calculamos as áreas de aglomerados subanormais dentro de cada município brasileiro. Não aprofundamos na questão 8 , que remete a análise local; no entanto, as estruturas aqui levantadas permitem repensar, em um mundo globalizado, as relações global-local.

Enfim, toda a análise exploratória convergiu para a modelização gráfica, constituindo-se em um processo de busca de relações entre os modelos estatísticos desenvolvidos em superfícies geométricas e o espaço geográfico delimitado em um território específico, no caso, o território brasileiro. Nesse processo, a comunicação é o resultado de síntese dos procedimentos anteriores. Destaca-se que, apesar de existir uma sutil hierarquia envolvendo análise exploratória, modelização espacial e comunicação cartográfica, essas etapas conformam-se às necessidades mediante a construção dos produtos cartográficos.

Para isso, foi fundamental efetuar os procedimentos metodológicos com base na teoria de Brunet (2001), que propõe que as estruturas fundamentais identificadas sejam representadas na forma de modelos gráficos, ou seja, as "chaves de leitura do território", representados e denominados "coremas", estruturas elementares que compõem o espaço geográfico. Os modelos, portanto, correspondem a um esforço de capturar os coremas, de representar um fragmento do mosaico do mundo. O raciocínio geográfico está constantemente buscando por essas formas sugestivas, por padrões espaciais e por conexões estruturais que deem sentido às situações geográficas e a eventos históricos. A proposta de Brunet, entretanto, diferencia-se pela necessidade de representar graficamente a relação entre estruturas, não se limitando à cartografia temática de distribuições espaciais de fenômenos particulares.

Assim, para retratar a situação geográfica no território brasileiro, foram analisados conjuntamente: 1) os dados de presença e dispersão dos casos de Covid-19; 2) os dados do sistema de transporte e circulação (rodovias, aeroportos); 3) as densidades técnica e demográfica já analisadas e mapeadas por Santos e Silveira (2001) e Therry e Mello (2008). A análise articulada permitiu 
a identificação de estruturas territoriais que condicionam a direção e a intensidade do processo pandêmico no Brasil, identificadas pela superposição das formas de dispersão com as formas das estruturas territoriais preexistentes. Sugerem, assim, interdependência, indicando uma existência mutuamente condicionada.

A partir da identificação dos coremas, ou seja, as próprias estruturas do real, são empregadas chaves gráficas que representam as relações e dão sentido à situação geográfica. Essas chaves podem se apresentar na forma de manchas, indicando contiguidade do fenômeno que se estende em todas as direções; em eixos, quando o fenômeno se estabelece em um ou dois sentidos; em pontos, quando um fenômeno se diferencia significativamente do seu entorno. Articuladamente, essas chaves podem representar uma quantidade significativa de formas e processos, representados de forma sintetizada.

Como já dissemos, o produto cartográfico resultante - um modelo gráfico, não é uma simplificação, mas uma síntese que procura capturar o essencial da situação em suas estruturas mais significativas e que são doadoras de sentido. $\mathrm{O}$ modelo gráfico apresentado destaca coremas ou estruturas do espaço indispensáveis para compreender e estudar a dinâmica da Covid-19 no território brasileiro. Não havendo softwares específicos que produzam automaticamente modelos gráficos territoriais - visto que isso dependerá do aporte teórico e metodológico construído pela investigação, é preciso empregar um software de desenho gráfico. Para a elaboração do modelo da Covid-19 apresentado na Figura 4, foi empregado o software Adobe Illustrator ${ }^{\circledR}$. Anterior a essas estruturas, utilizamos o ArcGIS 10.8 (Imagem, ESRI) e o software estatístico Minitab 16.

\section{Resultados e discussão}

O primeiro caso de Covid-19 foi registrado no território brasileiro no final do mês de fevereiro (Rodriguez-Morales et al., 2020). Na Figura 1 podemos visualizar o fenômeno se difundindo a partir das principais metrópoles do país, especialmente São Paulo, Rio de Janeiro, Vitória, Brasília, Salvador, Recife, Fortaleza, Belém, Manaus, Curitiba, Florianópolis e Porto Alegre. No estado de São Paulo e na região Norte do Brasil a velocidade de difusão foi grande, cobrindo grande parte do interior em menos tempo (predomínio de cores mais escuras). A faixa litorânea brasileira tem o início dos casos confirmados antes do restante do conjunto do território, evidenciando o processo de interiorização.

No processo de geração da superfície de densidade Kernel, a representação foi estabelecida com a aplicação de simbologia de mapa de calor de modo que represente a densidade relativa das taxas de casos confirmados presentes nas feições pontuais. A paleta de cores utilizada para essa representação cartográfica é conhecida como viridis inferno, a qual é recomendada para definir uma percepção visual uniforme em combinação com o fundo da representação cartográfica em preto. As tonalidades que variam de cores escuras para cores claras sobre o mapa com fundo escuro permitem o estímulo do pensamento visual para melhor compreensão dos padrões espaciais. Verifica-se maior densidade das taxas 


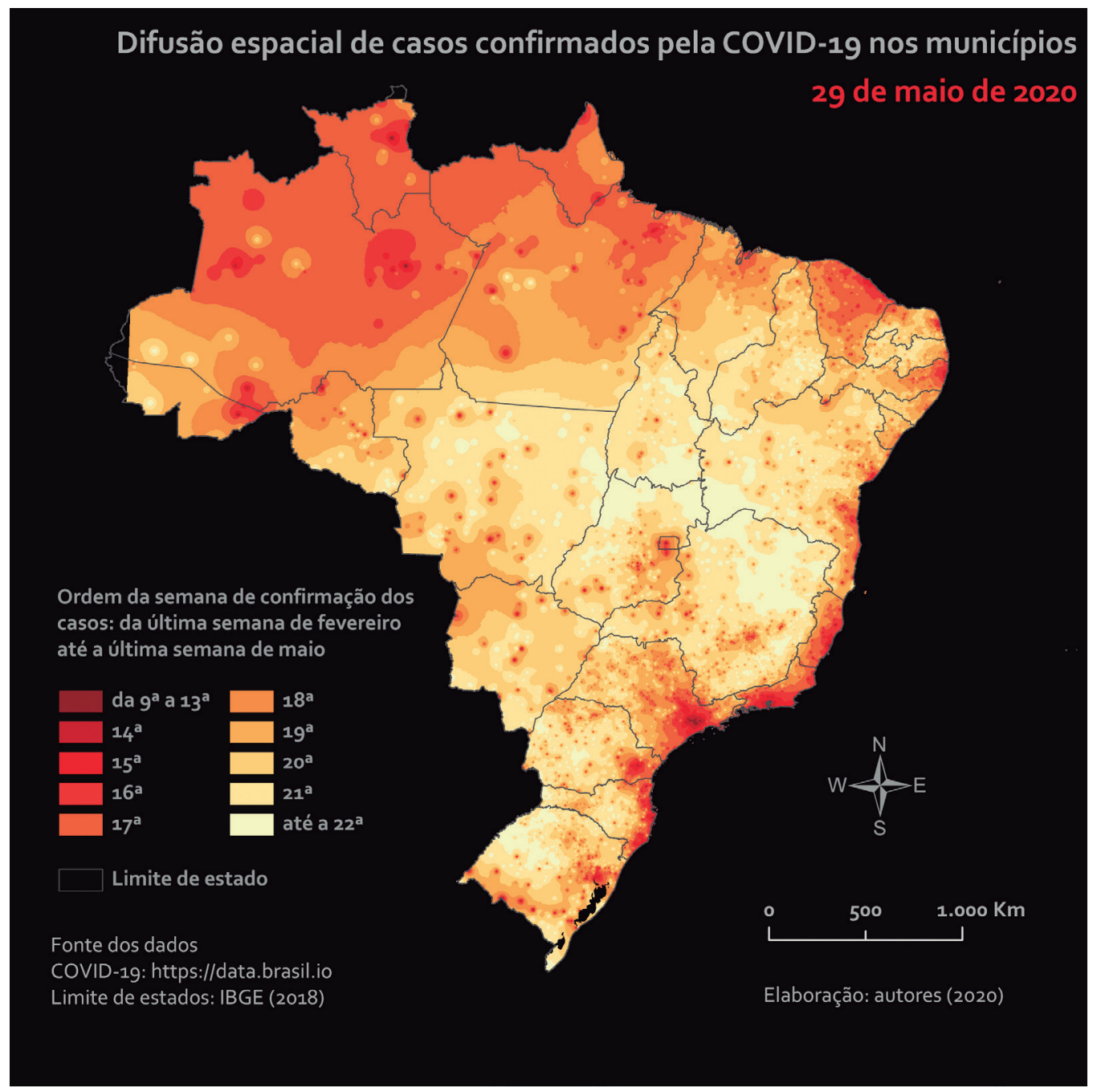

Fonte: Elaboração dos autores.

Figura 1 - Difusão espacial no território brasileiro.

de casos confirmados em algumas faixas nas regiões Norte e Nordeste, especialmente na área litorânea brasileira, e nas regiões Sudeste e Sul (Figura 2). Os espaços luminosos parecem se sobrepor as áreas de média densidade. 
A)

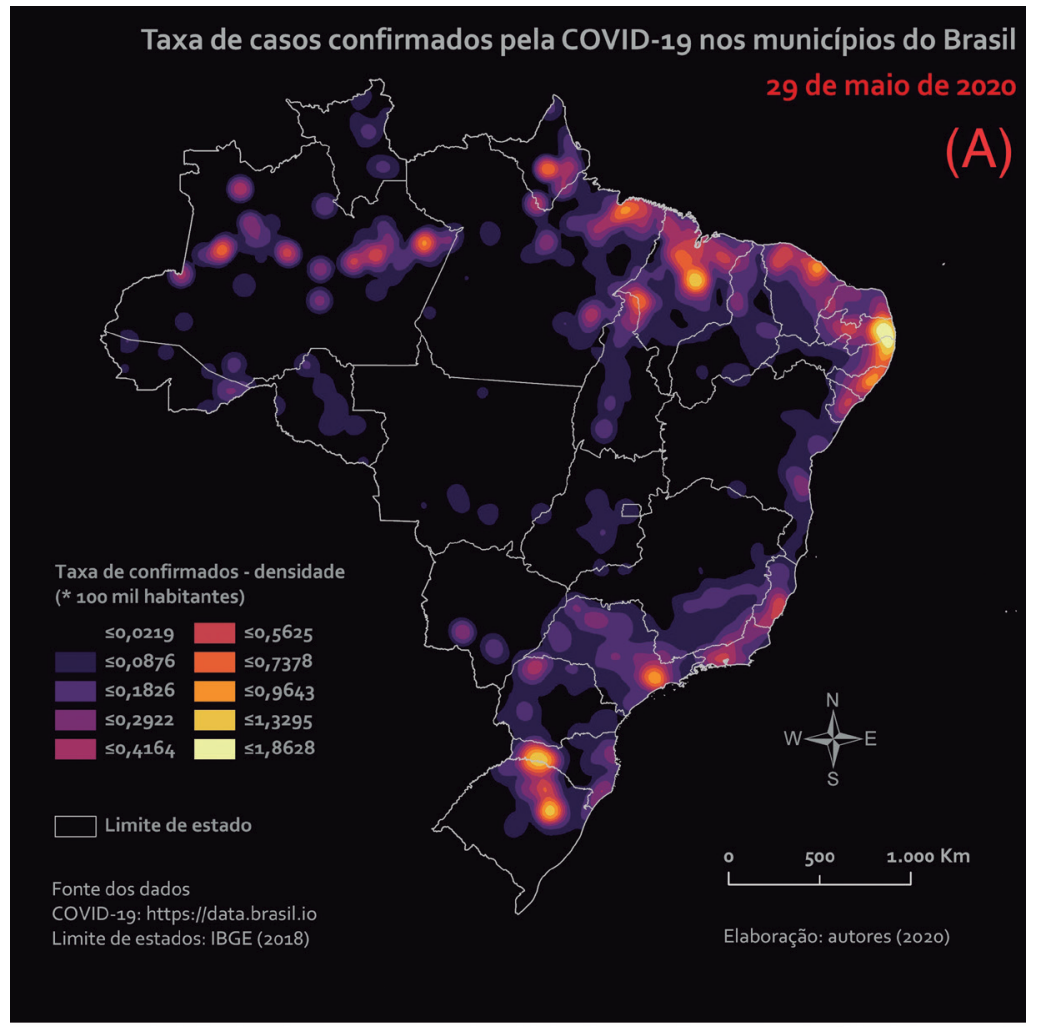

B)

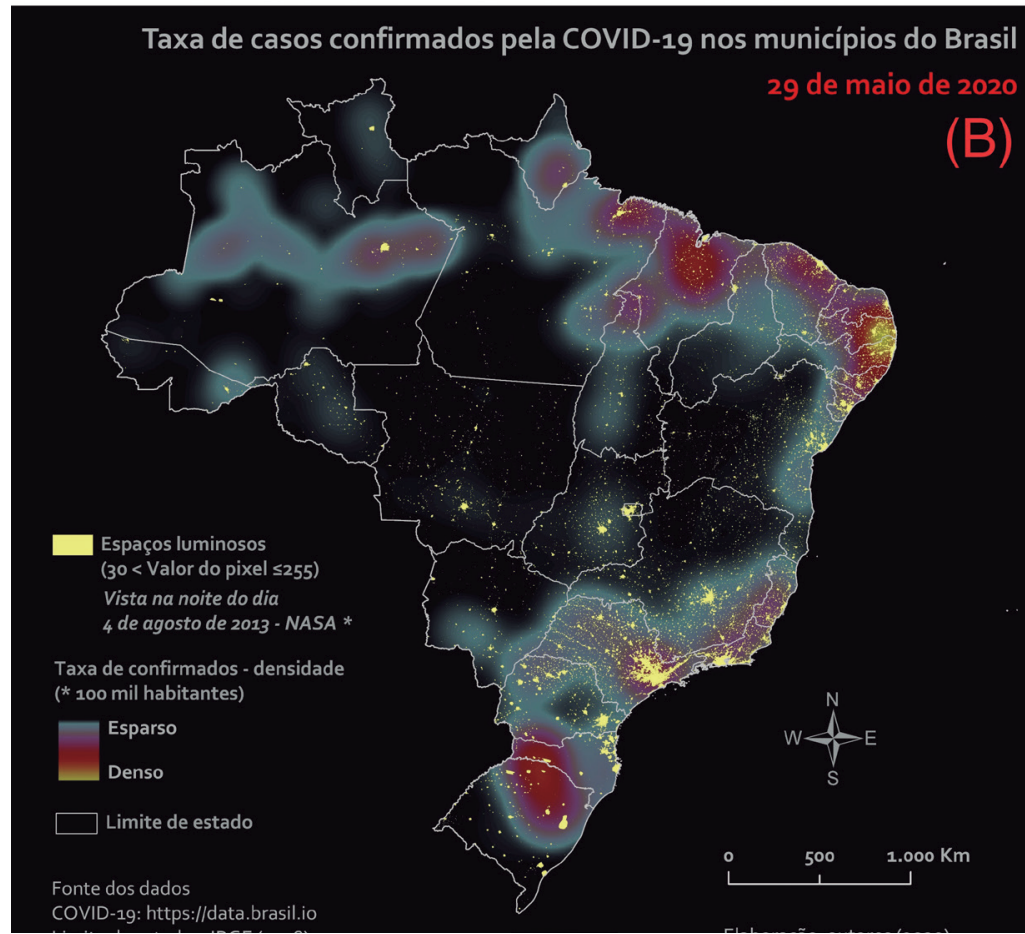

Fonte: Elaboração dos autores.

Figura 2 - Superfície de densidade (A) destaque aos núcleos de máxima densidade, (B) destaque aos locais de alta, média e baixa densidade. 
Por meio da Média do Vizinho Mais Próximo, obtivemos um índice de $0,66(\mathrm{Z}=-0,47)$, o que indica a presença significativa de aglomerados ( $\mathrm{p}$-valor $<$ $0,001)$. O valor da distância média observada foi de 17.886,4357 quilômetros.

Conforme os resultados representados na Figura 3, o índice de Moran foi de $0,109298(\mathrm{z}$-score $=67,25 ; \mathrm{p}$-valor $<0,001)$ o que indica a presença significativa de aglomerados ( $\mathrm{p}$-valor $<0,001)$. Observa-se que o Norte e o Nordeste do Brasil, este último sobretudo na faixa litorânea, são marcados por extensas áreas de agrupamentos com altas taxas de casos confirmados. Há também um cluster alto no Sul do Brasil, compreendendo o oeste do estado do Paraná em direção ao Rio Grande do Sul. De outra maneira, aglomerados com baixas taxas estão presentes em estados continentais brasileiros, com pequenos aglomerados de muito alto com padrão quase que de regular distribuição, que parecem indicar as centralidades regionais desses municípios.

Tendo isso em vista, encontramos correlação positiva de 0,54 para casos de Covid-19 e área de aglomerados subanormais, bem como correlação também positiva de 0,53 para óbitos de Covid-19 e áreas de aglomerados subanormais, ambas com significância estatística ( $\mathrm{p}$-valor $<0.000$ ). Em outras palavras, os casos de Covid-19 e de óbitos em decorrência desta enfermidade no território brasileiro crescem proporcionalmente ao aumento das áreas com aglomerados subanormais. Assim, atentamos para a vulnerabilidade do território frente à pandemia de Covid-19, uma vez que essa doença não ocorre de maneira homogênea pelo espaço (Dorn et al., 2020).

Fatores sociodemográficos têm sido associados com maiores taxas de infecção da doença (Lusignan et al., 2020) ou de óbitos em relação à Covid-19, incluindo maiores razões de chance para populações que vivem em ambientes menos favorecidos comparados aos mais favorecidos (Williamson et al., 2020; Cabrams; Szefler, 2020). Não se trata de questões relacionadas a fatores genéticos, mas de destacar a vulnerabilidade socioeconômica em que se encontram determinados grupos em detrimento de outros, considerando um país de dimensões continentais como o Brasil, com enorme diversidade sociocultural e desigualdades intrínsecas. 
A)

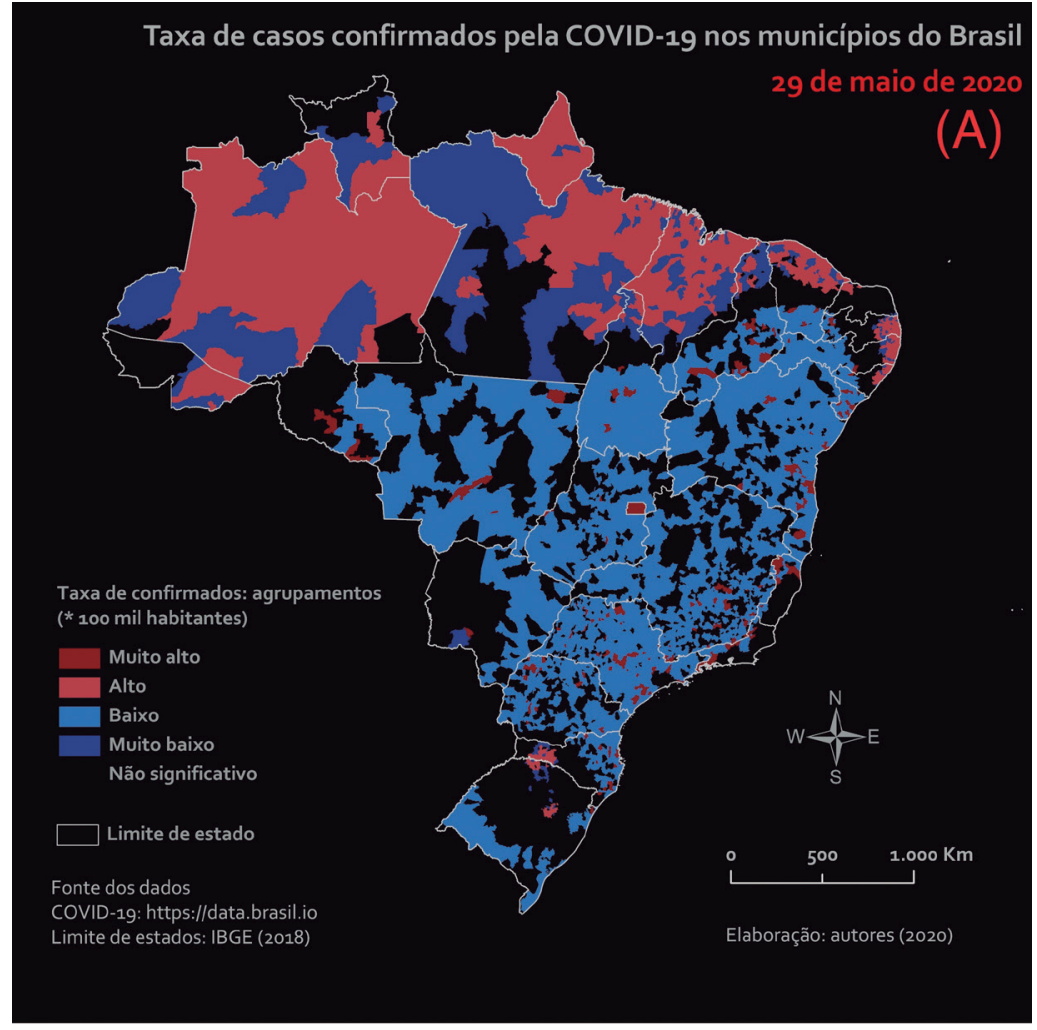

B)

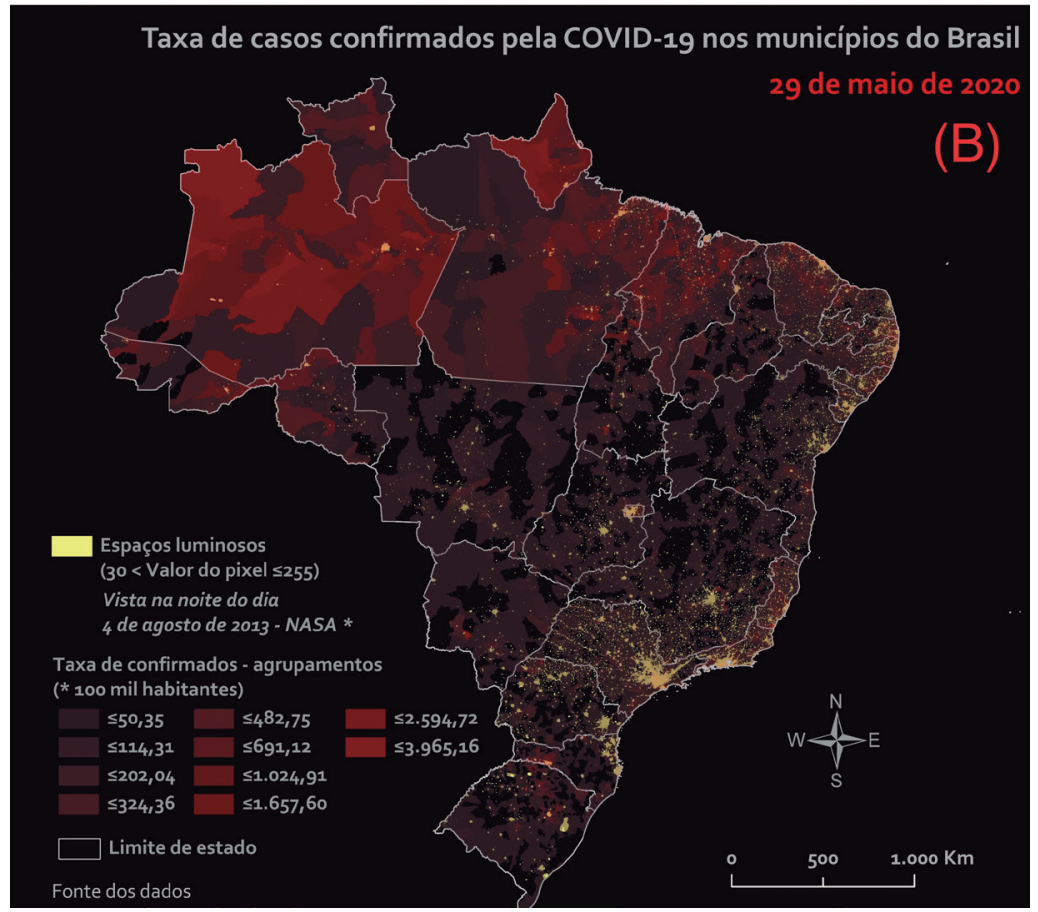

Fonte: Elaboração dos autores.

Figura 3 - Agrupamentos (A) destaque aos baixos e altos grupos, (B) destaque aos agrupamentos diversos. 
Finalmente, esses mapeamentos e técnicas estatísticas empregadas foram sistematizados na Figura 4. A dinâmica espacial da Covid-19 se deu ancorada em estruturas territoriais já conhecidas pelos geógrafos. Milton Santos e Maria Laura Silveira (2001), por exemplo, atentam para uma área de altas densidades, na qual os sistemas técnicos se dão de maneira contínua, como uma mancha, que foi denominada de Região Concentrada. Essa região inclui, atualmente, os estados das regiões Sul, grande parte da região Sudeste (à exceção do norte de Minas Gerais) e uma parte do Centro-Oeste. Fora da Região Concentrada, as densidades técnicas se apresentam na forma de eixos e pontos isolados no território. Hervé Thery (2008) identificou com clareza esses eixos. Eles se constituem basicamente em três: 1) um primeiro que conecta a Região Concentrada a Porto Velho, um desenho que acompanha a direção da BR-364, sobretudo; 2) um segundo que conecta a região concentrada a Belém, passando por Brasília, acompanhando a BR-153, sobretudo; e 3 ) um terceiro eixo que acompanha a faixa litorânea, correspondente ao povoamento inicial do país, onde estão as maiores densidades demográficas, econômicas e infraestruturais.

Em razão da grande diversidade do território brasileiro, ao analisar o processo de dispersão da Covid-19, é preciso considerar a transição do sistema rodoviário para o sistema hidroviário. Nos três eixos destacados o movimento de populações e produtos entre municípios se dá por via terrestre. Ao passar para o espaço amazônico, esse movimento se dá, basicamente, por meio de hidrovias. Há, portanto, no Brasil, dois sistemas de movimentos de pessoas que distinguem as regiões: um rodoviário e um hidroviário.

Ao destacar as áreas de aglomerados subanormais e terras indígenas demarcadas, quisemos garantir que a saúde não seja um subproduto do privilégio (Dorn et al., 2020) e que essas estruturas espaciais façam parte das discussões em saúde pública e que sejam sempre lembradas frente às medidas de enfrentamento da Covid-19 em território brasileiro.

O mapeamento e análise da Covid-19 no território permite constatar que sua evolução se dá estreitamente associada a essas estruturas territoriais, que são formas fixas associadas aos fluxos por elas conformadas. Uma vez considerado que o vírus é transmitido de pessoa a pessoa, os meios de transporte se constituem nas rotas preferenciais. Em razão dessas características, a geografia pode fornecer subsídios não somente ao diagnóstico das dinâmicas territoriais dos casos da doença, mas também para a realização de prognósticos que possam orientar as ações de saúde pública. 


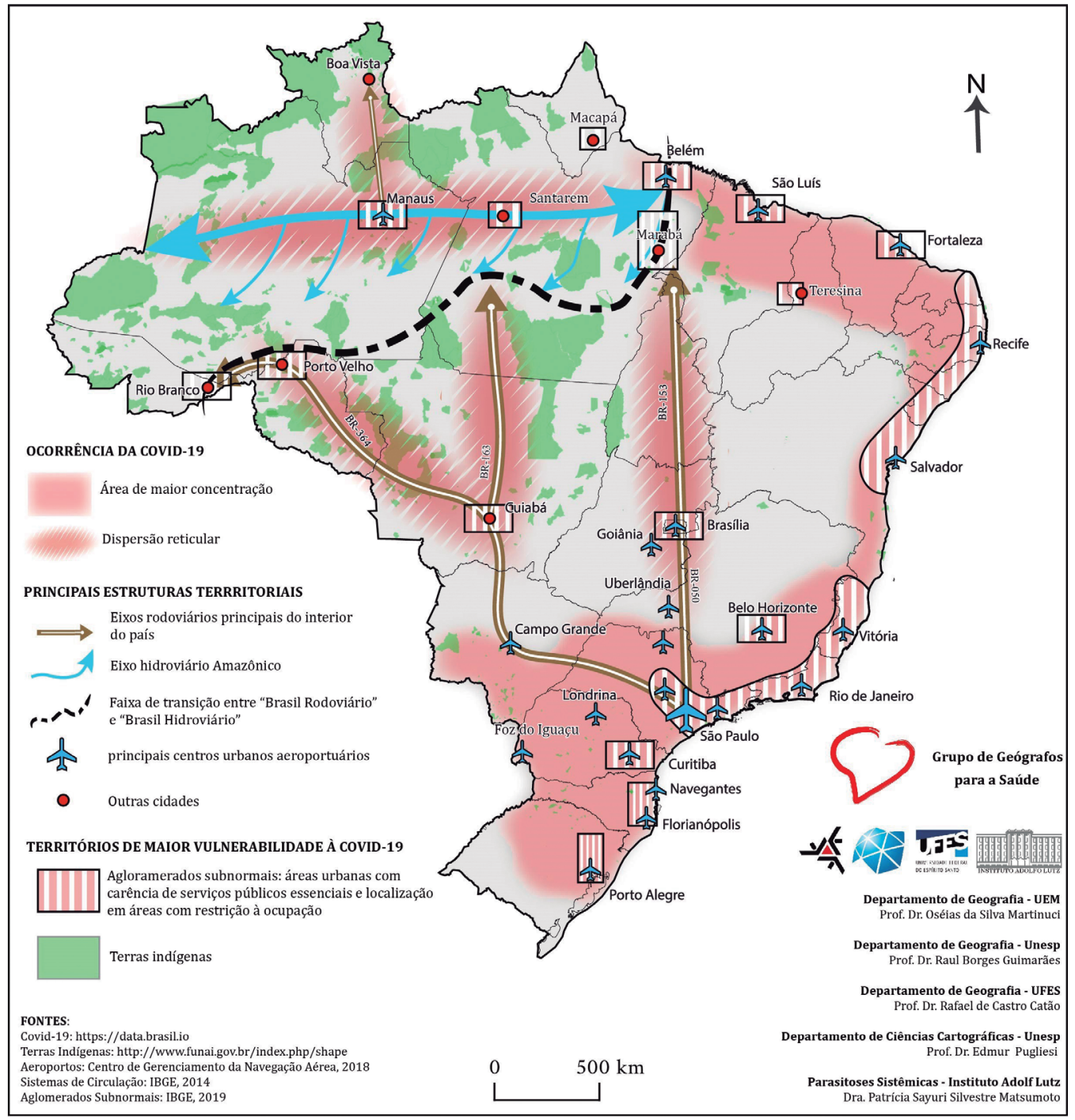

Fonte: Elaboração dos autores.

Figura 4 - Principais estruturas territoriais brasileiras da Covid-19.

\section{Considerações finais}

Por meio do processo de mapeamento desenvolvido neste trabalho, pôde-se observar que a dispersão do vírus pelo território brasileiro se deu a partir dos espaços de maior densidade de relações. A organização econômica do país modelou a direção, a temporalidade e a intensidade dos casos da Covid-19. As redes de transportes rodoviários de maior densidade de circulação constituíram-se nas rotas preferenciais. Essas redes estão intimamente relacionadas às dinâmicas econômicas que se traduzem em hierarquias territoriais, entre regiões e entre cidades. Nessas condições, a Covid-19, ao dispersar-se no território brasileiro, assumiu feições geográficas zonais, reticulares e pontuais. 
Assim, a análise exploratória, a modelização espacial e a comunicação cartográfica auxiliaram a compreensão da Covid-19, doença essa que exige esforços para além de uma análise simplificada do espaço geométrico. É por isso que os mapas produzidos não se constituíram no resultado da análise, mas no ponto de partida para o aprofundamento do raciocínio geográfico, referenciados em importantes teóricos da tradição geográfica, tais como Max Sorre, Torsten Hagerstrand, Roger Brunet, Brian Berry e Milton Santos. Dessa maneira, o olhar geográfico, assim como suas imagens gráficas nas mais diversas formas de representação (mapas, croquis, esboços etc.), pressupõe uma ideia de ordenação, estabelecida por uma sequência de princípios de raciocínio que conformam o conhecimento geográfico.

É por isso que entendemos que esses princípios do raciocínio geográfico são muito importantes para a compreensão da difusão espacial do novo coronavírus, guardada as especificidades desse fenômeno em cada lugar, como no caso da Amazônia. Somam-se a essas contribuições teorias como da visualização cartográfica e modelização, assim como da Gestalt; que complementam essa forma de pensar o mundo no processo de interação entre o mapeamento produzido e seu uso na leitura e interpretação do espaço socialmente produzido.

Nota

l Disponível em: <https://brasil.io/dataset/covid19/caso_full/>.

Referências

ABRAMS, E. M.; SZEFLER, J. S. Covid-19 and the Impact of Social Determinants of Health. The Lancet. Respiratory Medicine, 2020. Disponível em: <https://doi. org/10.1016/S2213-2600(20)30234-4>.

ALCÂNTARA, E. et al. Investigating spatiotemporal patterns of the Covid-19 in São Paulo State, Brazil. medRxiv. 2020. preprint <doi: https://doi.org/10.1101/2020.0 5.28.20115626>.

ANSELIN, L. Local Indicators of Spatial Association - LISA, Geographical Analysis, v.27, n.2, p.93-115, 1994.

BAUD, D. et al. Real estimates of mortality following Covid-19 infection. The Lancet Infectious Diseases. 2020. <DOI:https:// doi.org/10.1016/S1473-3099(20)30195$-\mathrm{X}>$.

BERRY, B. Approaches to regional analysis: Asynthesis. Annals of the Association of American Geographers, Chicago, p.1-7, 1964.<https://doi.org/10.1111/j.1467-8306.1964. tb00469.x>.

BROWN, L. Innovation Diffusion: a new perspective. New York: Methuen, 1981.

BRUNET, R. Des modèles em géographie? Sens d'une recherche. Bulletin de la Société Géographique de Liège, v.39, n.2, p.21-30, 2000. Disponível em: <https://popups.uliege.be:443/0770-7576/ index.php?id=2527>. 
BRUNET, R. Le dechiffrement du monde. Paris: Belin, 2001.

CÂMARA, G. et al. Análise espacial e geoprocessamento. In: DRUCK, S. et al. Análise Espacial de Dados Geográficos. Brasília: Embrapa, 2004a. p.31-56.

CÂMARA, G. et al. Análise espacial de dados geográficos. Brasília: Embrapa, 2004b. p.136-80.

CANADÁ. Focus on Wuban, China. The Canadian Trade Commissioner Service. Disponível em: <https://www.tradecommissioner.gc.ca/china-chine/market-facts-faits-sur-le-marche/ 96289.aspx?lang=eng>. Acesso em: 4 jun. 2020.

DORN, A. et al. Covid-19 Exacerbating Inequalities in the US. The Lancet, v.395, n.10232, p. 1243-4. 2020. <https://doi.org/10.1016/s0140-6736(20)30893-x>.

FERREIRA, M. C. Um modelo para formulação de perguntas geoespaciais em SIG, baseado na Matriz Geográfica de Berry (1964). Revista de Geografia da USP, v.26, p.252-69, 2013. https://doi.org/10.7154/RDG.2013.0026.0013.

FORTALEZA, C. M. B. et al. Elementary spatial structures and dispersion of Covid-19: health geography directing responses to public health emergency in São Paulo State. PRE Brazil. p.1-10, 2020. medRxiv: <https://doi.org/10.1101/2020.04.26. 20080895>.

GUIMARÃES, R. B.; CATÃO, R. C.; CASAGRANDE, B. Raciocínio geográfico e complexos patogênicos atuais: análise comparativa da Dengue e da Leishmaniose Tegumentar Americana. Confins [Online], v.37, 2018. <https://doi.org/10.4000/confins.15117>.

HÄGERSTRAND. T. Propagation of innovation waves. In: WAGNER, P.; MIKESSEL, M. (Ed.) Readings in cultural geography. Chicago: The Univerity of Chicago Press, 1962.

1968.

Inovation diffusion as an spatial process. Chicago: University of Chicago Press,

KOSIK, K. Dialética do concreto. 5.ed. Rio de Janeiro: Paz e Terra, 1989.

LUSIGNAN, S. et al. Risk Factors for SARS-CoV-2 among Patients in the Oxford Royal College of General Practitioners Research and Surveillance Centre Primary Care Network: A Cross- Sectional Study. The Lancet. Infectious Diseases 3099. 2020. <https://doi.org/10.1016/S1473-3099(20)30371-6>.

MACEACHREN, A. M. How Maps Work. Representation, Visualization, and Design. New York: Guilford, 2004.

MATSUMOTO, P. S. S. A geografia é uma forma de pensar: padrões espaciais e epidemiológicos da leishmaniose visceral em Araçatuba, Presidente Prudente e Votuporanga - SP, Brasil. Faculdade de Ciências e Tecnologia da Universidade Estadual Paulista "Júlio de Mesquita Filho" (FCT/UNESP). 2019. Disponível em: <https://repositorio. unesp.br/handle/11449/182323>.

MELLAN, T. et al. Report 21 Estimating Covid-19 cases and reproduction number in Brazil. Medrxiv., p.1-24. May 2020. <https://doi.org/10.1101/2020.05.09.20096701>.

MORILL, R. L. Waves of spatial diffusion. Journal of Regional Science, n.8, p.118,1968. <https://doi.org/10.1111/j.1467-9787.1968.tb01281.x>. 
RODRIGUES, W.; PRATA, D. P.; CAMARGO, W. Regional determinants of the expansion of COVID-19 in Brazil. MedRxiv. 2020. <https://doi.org/10.1101/2020.0 4.13.20063925>.

RODRIGUEZ-MORALES, A. J. et al. Covid-19 in Latin America: The Implications of the First Confirmed Case in Brazil. Travel Medicine and Infectious Disease., p.14778939, abr. 2020. <https://doi.org/10.1016/j.tmaid.2020.101613>.

ROJAS, L. I. La diferenciación territorial de la salud en la recuperación de los contextos. In: BARCELLOS, C. (Org.) A geografia e o contexto dos problemas de saúde. Rio de Janeiro: Abrasco, 2008. p.87-106.

Geografia e saúde: o antigo, o novo e as dívidas. In: GURGEL, H.; BELLE, N. (Org.) Geografia e Saúde: Teoria e Método na Atualidade. Brasília: Universidade de Brasília, 2019.

SANTOS, M. A natureza do espaço. 4.ed. São Paulo: Edusp, 2002.

Por uma outra globalização. 15.ed. São Paulo: Record, 2008.

SANTOS, M.; SILVEIRA, M. L. O Brasil: Território e sociedade no início do século XXI. São Paulo: Record, 2001.

SILVEIRA, M. L. Uma situação geográfica: do método à metodologia. Revista Território, v.4, n.6, p.21-28, jan./jun. 1999.

SILVERMAN, B. W. Density Estimation for Statistics and Data Analysis. London: Chapman \& Hall/CRC, 1986.

SORRE, M. Complexes pathogènes et géographie médicale. Annales de Géographie, n.235, p.1-18, jan. 1933.

1957.

Rencontres de la Géographie et de la Sociologie. Paris: Marcel Rivière et Cie.,

Principes de cartographie applique a l'écologie humaine. Social Science é $\mathrm{Me}$ dicine, v.12, D, p. 238-50, 1978.

THÉRY, H. Modelagem gráfica para a análise regional: um método. Geousp, São Paulo, n.15, p.179-88, 2004.

THERY, H.; MELlO, N. A. Atlas do Brasil. São Paulo: Edusp, 2008.

WILLIAMSON, E. et al. OpenSAFELY: Factors Associated with Covid-19-Related Hospital Death in the Linked Electronic Health Records of 17 Million Adult NHS Patients. MedRxiv. 2020. <https://doi.org/10.1017/CBO9781107415324.004>.

RESUMO - Para compreender como a Covid-19 se distribui pelo espaço brasileiro, os geógrafos utilizam-se do raciocínio geográfico apoiados em técnicas de mapeamento e representações espaciais. Nesse sentido, propomos aqui uma série de procedimentos para compreender a doença no espaço, primeiramente com a exploração e descrição dos dados, a análise espacial e a síntese por meio da modelização gráfica, partindo em seguida para a comunicação cartográfica. Como efeito, esperamos que esse caminho teórico e metodológico possa balizar a criação de uma imagem de mundo capaz de responder a demandas mais urgentes postas pela pandemia, ao mesmo tempo criar reflexões sobre como a produção do espaço atual cria vulnerabilidades nesta globalização perversa. 
PALAVRAS-CHAVE: Raciocínio geográfico, Análise exploratória, Modelagem, Visualização, Covid-19.

ABSTRACT - To understand how covid-19 is distributed in Brazil, geographers use geographical reasoning supported by mapping techniques and spatial representation. We thus propose here a series of procedures to understand the disease in the geographic space, first with an exploration and descriptive analysis of the data, a spatial analysis and a synthesis through graphical modeling, followed by a cartographic communication. As a result, we hope that this theoretical and methodological path may guide the creation of a world image capable of responding to the most urgent demands posed by the pandemic, and concomitantly create reflections on how the production of geographical space creates vulnerabilities in this perverse globalization.

KEYWORDS: Geographic reasoning, Exploratory analysis, Modeling, Visualization, Covid-19.

Raul Borges Guimarães é professor titular do Departamento de Geografia da Faculdade de Ciências e Tecnologia da Unesp. @ - raul.guimaraes@unesp.br /

https://orcid.org/0000-0002-9925-5374.

Rafael de Castro Catão é doutor, professor adjunto do Departamento de Geografia da Universidade Federal do Espírito Santo. @ - rafael.catao@ufes.br / https://orcid.org/0000-0003-2837-0364.

Oséias da Silva Martinuci é doutor, professor adjunto do Departamento de Geografia da Universidade Estadual de Maringá. @ - oseiasmartinuci@yahoo.com /

https://orcid.org/0000-0002-5206-4829.

Edmur Azevedo Pugliesi é doutor, professor do Departamento de Ciências Cartográficas da Faculdade de Ciências e Tecnologia da Unesp. @ - edmur.pugliesi@unesp.br / https://orcid.org/0000-0003-3426-6619.

Patrícia Sayuri Silvestre Matsumoto é pós-doutoranda da Parasitoses sistêmicas, Instituto Adolfo Lutz Central (SP). @ - pamatsumot@gmail.com /

https://orcid.org/0000-0001-7205-7557.

Recebido em 6.6.2020 e aceito em 25.6.2020.

I, IV Faculdade de Ciências e Tecnologia, Universidade Estadual Paulista "Júlio de Mesquita Filho", Presidente Prudente, São Paulo, Brasil.

${ }^{\text {II }}$ Departamento de Geografia da Universidade Federal do Espírito Santo, Vitória, Espírito Santo, Brasil.

III Universidade Federal de Maringá, Maringá, Paraná, Brasil.

v Instituto Adolfo Lutz Central, São Paulo, Brasil. 
